\title{
The Safety and Feasibility of Enhanced Recovery after Surgery in Patients Undergoing Pancreaticoduodenectomy: An Updated Meta-Analysis
}

\author{
You-Meng Sun, Ying Wang, Yi-Xin Mao, and Wei Wang \\ The First Affiliated Hospital, Zhejiang University School of Medicine, Hangzhou, 310003 Zhejiang Province, China \\ Correspondence should be addressed to Wei Wang; wangw2005@zju.edu.cn
}

Received 2 January 2020; Accepted 24 March 2020; Published 9 May 2020

Academic Editor: Daithi Heffernan

Copyright ( $\odot 2020$ You-Meng Sun et al. This is an open access article distributed under the Creative Commons Attribution License, which permits unrestricted use, distribution, and reproduction in any medium, provided the original work is properly cited.

\begin{abstract}
Background. Enhanced recovery after surgery (ERAS) is a multimodal, multidisciplinary, evidence-based approach to care for surgical patients and aims at optimizing the perioperative management and outcomes. The ERAS approach was first implemented in colorectal surgery patients; however, the reported applications in pancreatoduodenectomy patients are limited. In recent years, studies on ERAS for patients undergoing pancreaticoduodenectomy have been published. The accumulation of new randomized controlled trials and high-quality case-control studies stimulated us to update the analysis. Our study comprehensively collected data to provide the best evidence summary for the clinic. Aim. To evaluate the safety and feasibility of enhanced recovery after surgery in the perioperative management of pancreatoduodenectomy patients. Methods. A systematic literature search of PubMed, Embase, and the Cochrane Library was performed up to July 2019. All randomized controlled trials and case-control studies that applied ERAS for patients undergoing pancreaticoduodenectomy were considered for inclusion in this study. The patients were divided into two groups: patients who received the ERAS perioperative management approach were defined as the ERAS group and patients who received the traditional perioperative management approach were defined as the control group. All statistical analyses were conducted using the Revman5.3 software, and the outcomes were calculated as odds ratios or weighted mean differences with their corresponding 95\% confidence intervals. A funnel plot was created to assess publication bias. Subgroup and sensitivity analyses were performed to explore the sources of heterogeneity. Results. A total of 20 studies involving 3613 patients (1914 patients in the ERAS group vs. 1699 patients in the control group) were included in this study. Among the 20 studies, 4 were randomized controlled trials, and 16 were case-control studies. The overall postoperative complication rate was significantly lower in the ERAS group (OR $=0.62,95 \% \mathrm{CI}$ : $0.53-0.74, P<0.00001)$ than in the control group. In addition, the minor complication rate (Clavien-Dindo I-II) was also lower in the ERAS group $(\mathrm{OR}=0.70,95 \% \mathrm{CI}$ : 0.58-0.86, $P=0.0005)$. The patients in the ERAS group had a lower incidence of delayed gastric emptying $(\mathrm{OR}=0.51,95 \% \mathrm{CI}$ : $0.42-0.63, P<0.00001)$ and shorter length of hospital stay (WMD $=-4.27,95 \% \mathrm{CI}:-4.81 \sim-3.73, P<0.00001)$ than in the control group. The rates of pancreatic fistula (regardless of Grade $\mathrm{A} / \mathrm{B} / \mathrm{C}$ ), wound infections, abdominal abscesses, readmission, reoperation, and morbidity were not significantly different between the two groups. Conclusion. The ERAS approach is safe and effective in the perioperative management of patients undergoing pancreaticoduodenectomy and helps to accelerate the postoperative recovery and improve prognosis.
\end{abstract}

\section{Introduction}

Enhanced recovery after surgery (ERAS) was first proposed by Kehlet [1] from the University of Copenhagen in Denmark in 1997. ERAS is a multimodal, multidisciplinary, evidence-based approach to care for surgical patients and aims at optimizing the perioperative management and outcomes. The aim of this approach is to alleviate the patient's surgical stress response, reduce postoperative complications, promote functional recovery, shorten the length of hospital stay, and achieve rapid recovery [2]. The ERAS approach was first implemented in colorectal surgery patients [3] and 
has now been widely applied all over the world. Different ERAS guidelines or consensuses have been published in multiple areas such as rectal/pelvic surgery [4], pancreaticoduodenectomy [5], radical cystectomy [6], gastrointestinal surgery [7], lung surgery [8], colorectal surgery [9], gynecologic/oncology [10], cesarean delivery [11], and cardiac surgery [12]. Pancreaticoduodenectomy, also known as the Whipple procedure, is the only potentially curative intervention for malignant tumors of the pancreas and duodenal ampulla.

Pancreaticoduodenectomy is often considered one of the most challenging operations in general surgery, and it takes a long time for patients to recover. In recent years, a series of studies on ERAS, which has been widely applied in patients undergoing pancreaticoduodenectomy, have been published $[13,14]$. These studies suggested that implementation of ERAS programs in patients undergoing pancreaticoduodenectomy is a safe and effective approach to promote postoperative recovery. The accumulation of new randomized controlled trials and high-quality case-control studies stimulated us to update the analysis. Our study comprehensively collects data to provide the best evidence summary for the clinic.

\section{Materials and Methods}

2.1. Literature Search. A systematic literature search of PubMed, Embase, and the Cochrane Library was performed to collect randomized controlled trials or case-control studies that applied the ERAS approach in the perioperative management of patients undergoing pancreaticoduodenectomy, and all of the studies were performed from the inception of the database to July 2019. The search terms and relative variants were as follows: "Pancreaticoduodenectomy," "Pancreatectomy," "Duodenopancreatectomy," "ERAS," "enhanced recovery after surgery," "FTS," "fast track surgery," "accelerated recovery surgery," "rapid recovery surgery," "clinical pathway," and "critical pathway." The PubMed search strategy is detailed in Table S1.

\subsection{Inclusion Criteria}

(1) Type of study: randomized controlled trials or casecontrol studies, with the language limited to English

(2) Participants: patients aged $\geq 18$ years who underwent elective pancreaticoduodenectomy or pyloruspreserving pancreaticoduodenectomy and patients who underwent a Whipple procedure, regardless of sex or nationality

(3) Interventions: patients who received the ERAS perioperative management approach defined as the experimental group and patients who received the traditional perioperative management approach defined as the control group. According to the guidelines [5] for the perioperative care of pancreaticoduodenectomy patients issued in 2012, at least 9 of 27 recommendation items should be implemented in the ERAS group.

\subsection{Exclusion Criteria}

(1) Full text of the article was not available

(2) Republished

(3) Focus on palliative surgery, emergency surgery, or laparoscopic pancreaticoduodenectomy

(4) According to the MINORS [15] scoring standard, the study quality score was lower than 13 points

(5) Unextractable useful outcomes.

2.4. Data Extraction and Quality Assessment. Data were extracted by two investigators independently, the following information was extracted from each eligible study: name of the author, year of publication, country, study design, the number of patients in the ERAS group and control group, the total sample size, demographic data, type of surgery, interventions, outcomes, and so on. Two investigators independently evaluated the quality of the articles, and any differences were unified through discussion and a consult with a third investigator. The quality of the randomized controlled trials was evaluated by the Cochrane risk assessment tool and the quality of the case-control studies were evaluated with the methodological index for nonrandomized controlled studies [15].

2.5. Outcomes of Interest. The primary outcome was the overall postoperative complications, and the secondary outcomes are the rates of pancreatic fistula, delayed gastric emptying, incision infections, abdominal abscesses, readmission, reoperation, and mortality as well as the length of hospital stay. Pancreatic fistula [16] was defined using the International Pancreatic Fistula Study Group (ISGPF) guidelines and was described as a drain output of any measurable volume of fluid on or after postoperative day 3 with an amylase content greater than 3 times the serum amylase activity. Delayed gastric emptying [17] was defined according to the International Study Of Pancreatic Surgery (ISGPS) as the need to maintain a nasogastric tube (NGT) for $>3 \mathrm{~d}$, postoperative vomiting for 3 days with a NGT or for 7 days while not being able to tolerate a solid diet. The length of hospital stay referred to the time from the date of surgery to the date of discharge. The overall postoperative complications included any complications within 30 days from surgery to discharge, and the severity was graded by the Clavien-Dindo system [18] into minor complications (Grades I-II) and moderate and major complications (Grades III-IV). Readmission was defined as a readmission within 30 days of discharge. Reoperation was defined as a reoperation required for patients with complications or for other reasons within 30 days after discharge. Mortality was defined as death within 30 days from surgery to discharge.

2.6. Statistical Analysis. Statistical analysis was performed with the Revman5.3 software, and the outcomes were calculated as odds ratios (ORs) or weighted mean differences (WMDs) with their corresponding 95\% confidence intervals (CIs). The heterogeneity between studies was analyzed by 


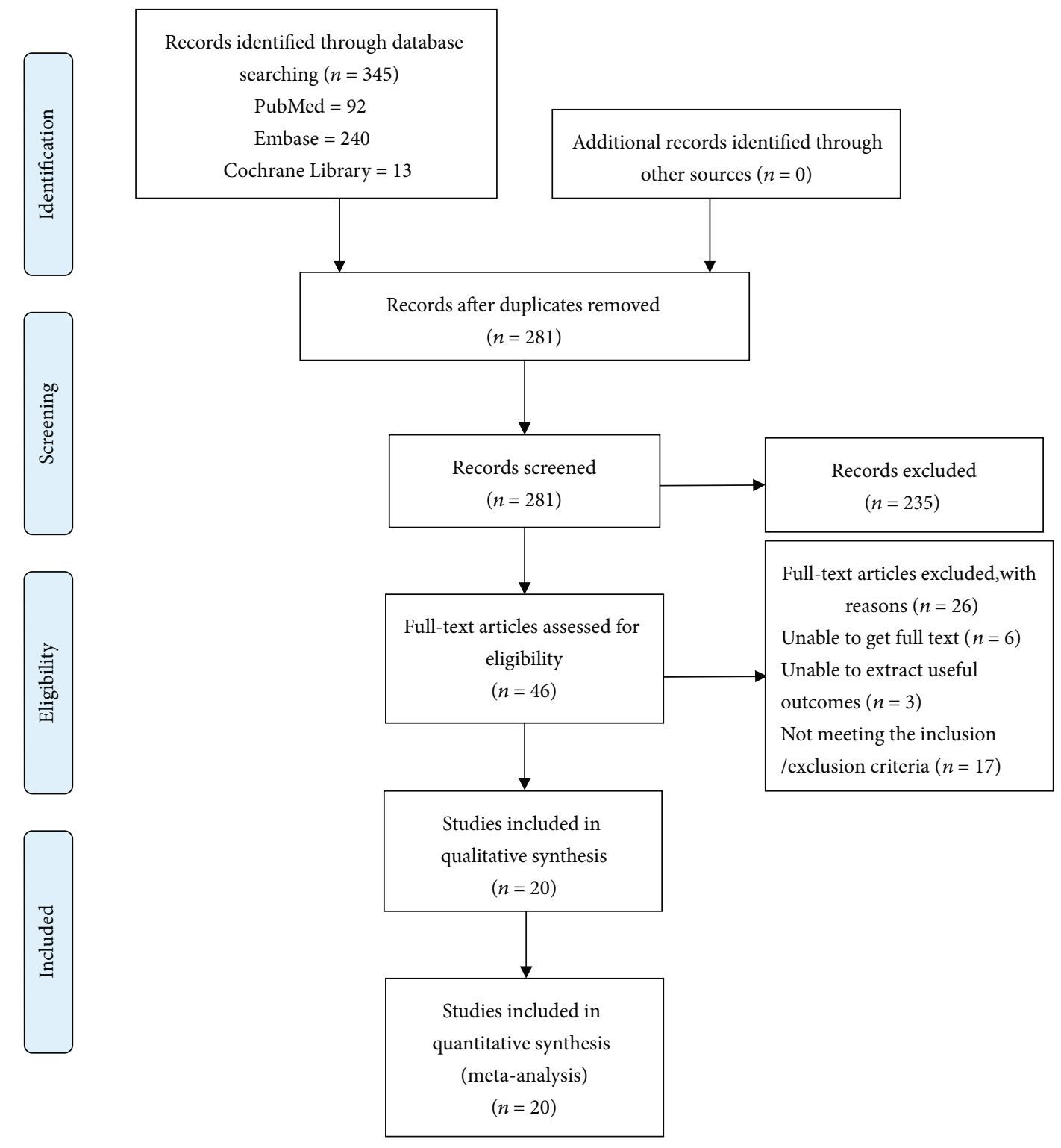

FIGURE 1: PRISMA flow diagram of the included studies eligible for meta-analysis.

the chi-squared test, with the test level being $\alpha=0.05$, and $I^{2}$ was used to measure the heterogeneity. Subgroup analyses were performed by separately analyzing only the Western countries, Eastern countries, case-control studies, and randomized controlled trials to explore the potential sources of heterogeneity. The overall postoperative complications were taken as the outcome, and sensitivity analyses were carried out by excluding one study in each round. In addition, a funnel plot was created to assess the publication bias based on the incidence of postoperative complications, pancreatic fistula, delayed gastric emptying, and mortality.

\section{Results}

3.1. Study Selection. According to the previous search strategy, a total of 345 records were retrieved from the online database up to July 2019. After removing the duplicates,
281 records remained, and 235 records were excluded by reviewing the title and abstract. After reading the remaining 46 records carefully, 26 records were removed for many reasons. Ultimately, 20 full-text studies [19-38] met the study inclusion criteria and were incorporated in this metaanalysis. A flow chart of the inclusion criteria to determine studies suitable for this meta-analysis is as follows (Figure 1). A total of 3613 patients (1914 patients in the ERAS group vs. 1699 patients in the control group) were involved, and among the 20 studies, 4 were randomized controlled trials [33, 35, 37, 38], and 16 were case-control studies $[19-32,34,36]$. The characteristics and quality assessments of the included studies are summarized in Table 1, and the demographics of the included studies are shown in Table S2.

3.2. Perioperative Interventions in the ERAS Group. An international working group, the European Association for 
TABLE 1: Characteristics and quality assessment of the included studies.

\begin{tabular}{|c|c|c|c|c|c|c|c|}
\hline \multirow{2}{*}{ Study } & \multirow{2}{*}{ Year } & \multirow{2}{*}{ Country } & \multirow{2}{*}{ Study design } & \multicolumn{2}{|c|}{ Sample size } & \multirow{2}{*}{ Total } & \multirow{2}{*}{ MINORS score } \\
\hline & & & & ERAS group & Control group & & \\
\hline Kennedy et al. & 2007 & United States & Case-control study & 91 & 44 & 135 & $15 / 24$ \\
\hline Vanounou et al. & 2007 & United States & Case-control study & 145 & 64 & 209 & $13 / 24$ \\
\hline Balzano et al. & 2008 & Italy & Case-control study & 252 & 252 & 504 & $15 / 24$ \\
\hline Abu Hilal et al. & 2013 & Britain & Case-control study & 20 & 24 & 44 & $14 / 24$ \\
\hline Kobayashi et al. & 2014 & Japan & Case-control study & 100 & 90 & 190 & $13 / 24$ \\
\hline Braga et al. & 2014 & Italy & Case-control study & 115 & 115 & 230 & $18 / 24$ \\
\hline Coolsen et al. & 2014 & Netherlands & Case-control study & 86 & 97 & 183 & $15 / 24$ \\
\hline Shao et al. & 2015 & China & Case-control study & 325 & 310 & 635 & $14 / 24$ \\
\hline Sutcliffe et al. & 2015 & Britain & Case-control study & 65 & 65 & 130 & $14 / 24$ \\
\hline Williamsson et al. & 2015 & Sweden & Case-control study & 50 & 50 & 100 & $16 / 24$ \\
\hline Morales Soriano et al. & 2015 & Spain & Case-control study & 41 & 44 & 85 & $16 / 24$ \\
\hline Joliat et al. & 2015 & Switzerland & Case-control study & 74 & 87 & 161 & $15 / 24$ \\
\hline Bai et al. & 2016 & China & Case-control study & 124 & 63 & 187 & $15 / 24$ \\
\hline Zouros et al. & 2016 & Greece & Case-control study & 75 & 50 & 125 & $16 / 24$ \\
\hline Deng et al. & 2017 & China & $\mathrm{RCT}$ & 76 & 83 & 159 & * \\
\hline Su et al. & 2017 & China & Case-control study & 31 & 31 & 62 & $15 / 24$ \\
\hline Takagi et al. & 2019 & Japan & RCT & 37 & 37 & 74 & * \\
\hline Perinela et al. & 2019 & France & Case-control study & 47 & 30 & 77 & $19 / 24$ \\
\hline Lavu et al. & 2019 & United States & RCT & 37 & 39 & 76 & ${ }^{*}$ \\
\hline Hwang et al. & 2019 & Korea & RCT & 123 & 124 & 247 & * \\
\hline
\end{tabular}

RCT: randomized controlled trial; MINORS: methodological index for nonrandomized studies. ${ }^{*}$ Unconformity to MINORS score criteria.

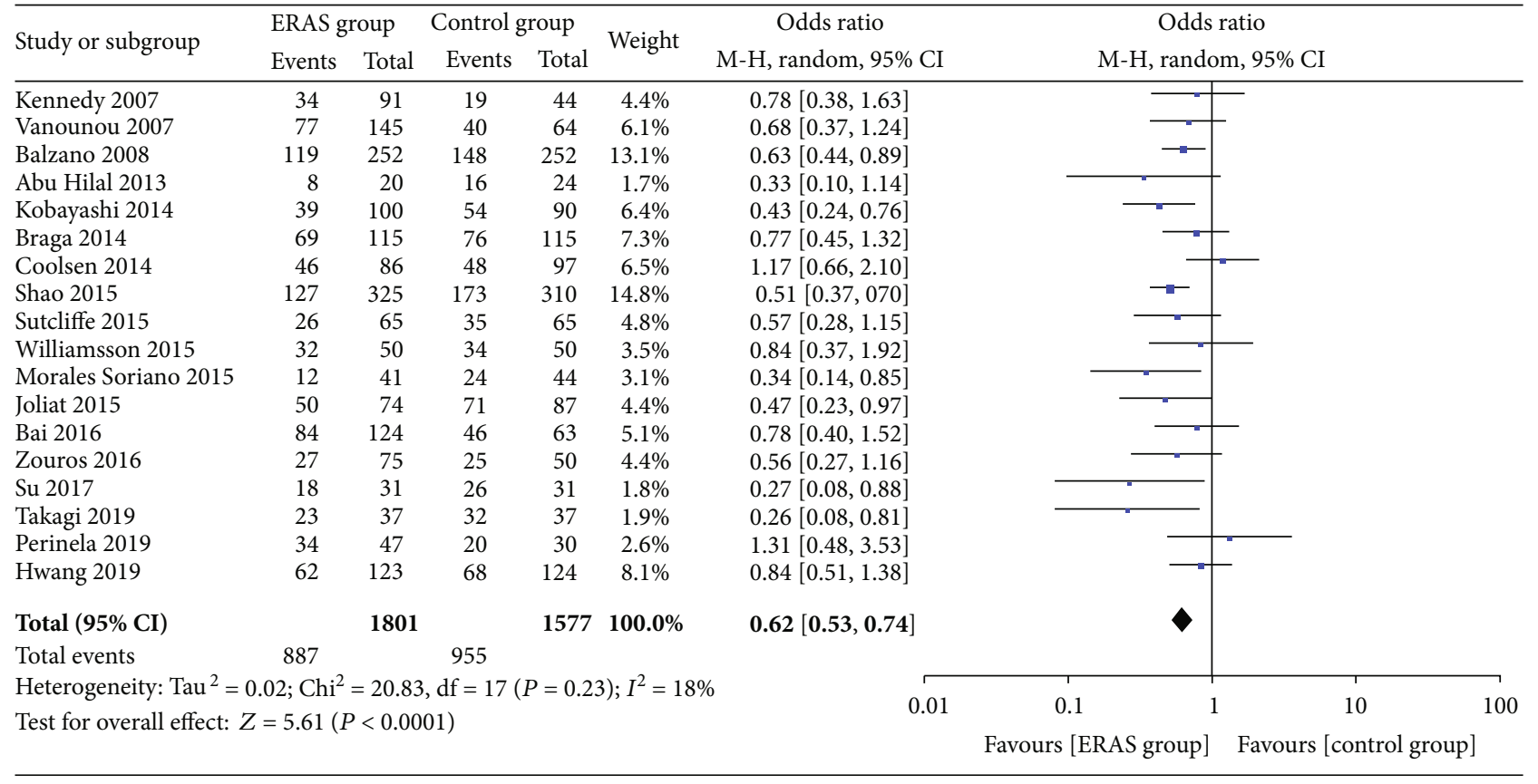

FIgURE 2: Forest plots demonstrating the outcomes of overall postoperative complications.

Clinical Nutrition and Metabolism, constructed an ERAS Society recommendation in 2012 [5] and provided a comprehensive, evidence-based framework that aims at optimizing perioperative care for pancreatoduodenectomy patients. The evidence and recommendations were classified according to the GRADE system, and the quality of the evidence 


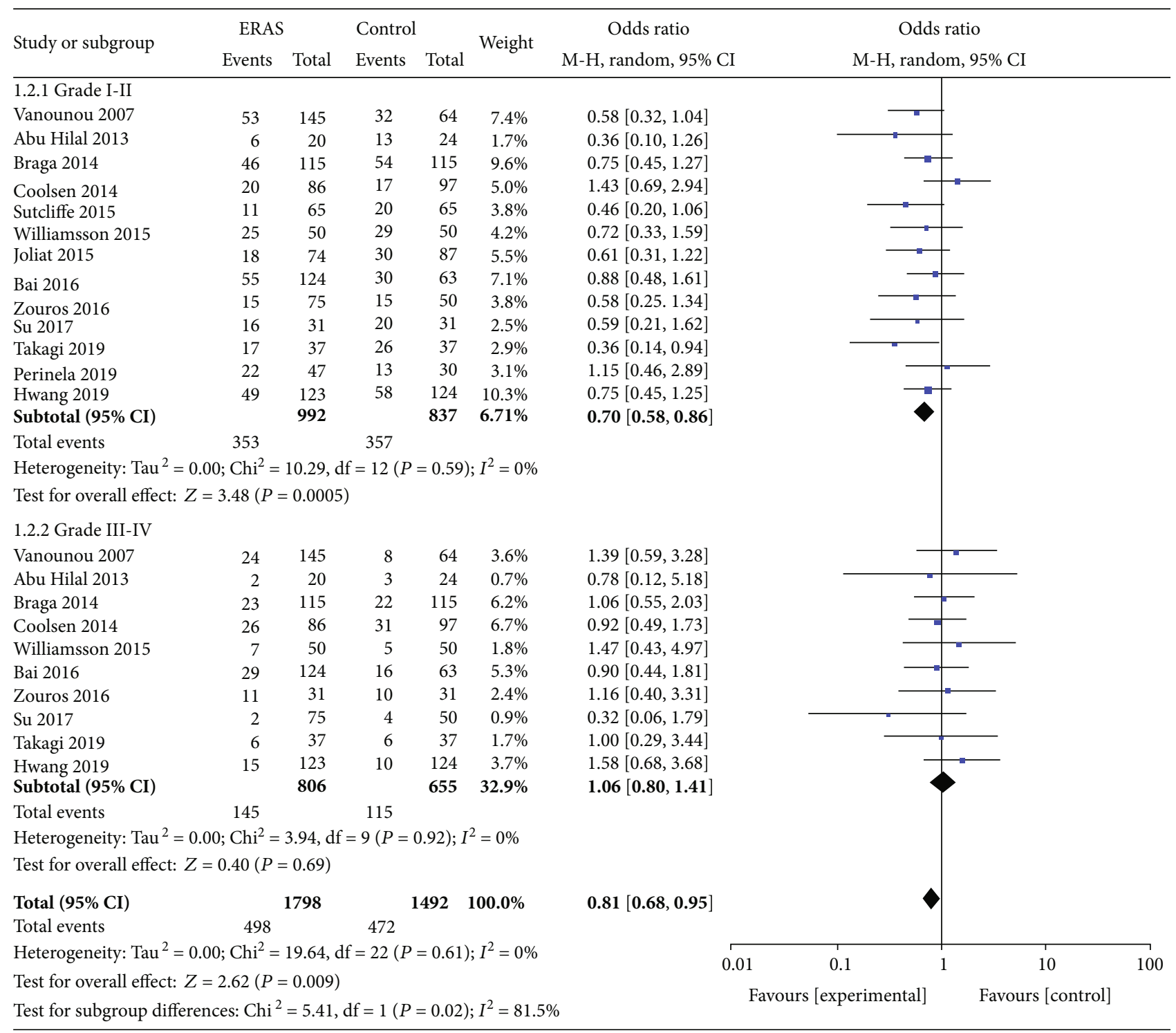

FIGURE 3: Forest plots demonstrating the outcomes of overall postoperative complications based on the classification of Clavien-Dindo.

was divided into four levels: "high," "moderate," "low," or "very low." The recommendations were graded as "strong" or "weak." The following 27 items were included: (1) preoperative counseling, (2) perioperative biliary drainage, (3) preoperative smoking and alcohol consumption, (4) preoperative nutrition, (5) perioperative oral immunonutrition (IN), (6) oral bowel preparation, (7) preoperative fasting and preoperative treatment with carbohydrates, (8) preanesthetic medication, (9) antithrombotic prophylaxis, (10) antimicrobial prophylaxis and skin preparation, (11) epidural analgesia, (12) intravenous analgesia, (13) wound catheters and transversus abdominis plane block, (14) postoperative nausea and vomiting (PONV), (15) incisions, (16) avoiding hypothermia, (17) postoperative glycaemic control, (18) nasogastric intubation, (19) fluid balance, (20) perianastomotic drain, (21) somatostatin analogues, (22) urinary drainage, (23) delayed gastric emptying (DGE), (24) stimulation of bowel movements, (25) postoperative artificial nutrition, (26) early and scheduled mobilization, and (27) audits. Among the included studies, 17 articles [22-38] that were published after 2012 had a high compliance rate with the interventions of different ERAS elements, and 3 articles [19-21] that were issued before 2012 met the standards of the interventions that applied more than 9 items and were also considered in our meta-analysis. The detailed elements of the ERAS approach of each study are shown in Table S3.

3.3. Quality Assessment of the Included Studies. Four randomized controlled trials [33, 35, 37, 38] all mentioned the generation of random sequences, one [37] of them referred to the grouping method, and the other [35] described the loss to follow-up. None of the subjects, intervention implementers, or outcome measure evaluators were blinded in these studies. The quality grades were Bs for all of the included randomized controlled trials. The MINORS scores of 16 casecontrol studies $[19-32,34,36]$ were $\geq 13$ points. The bias risk 


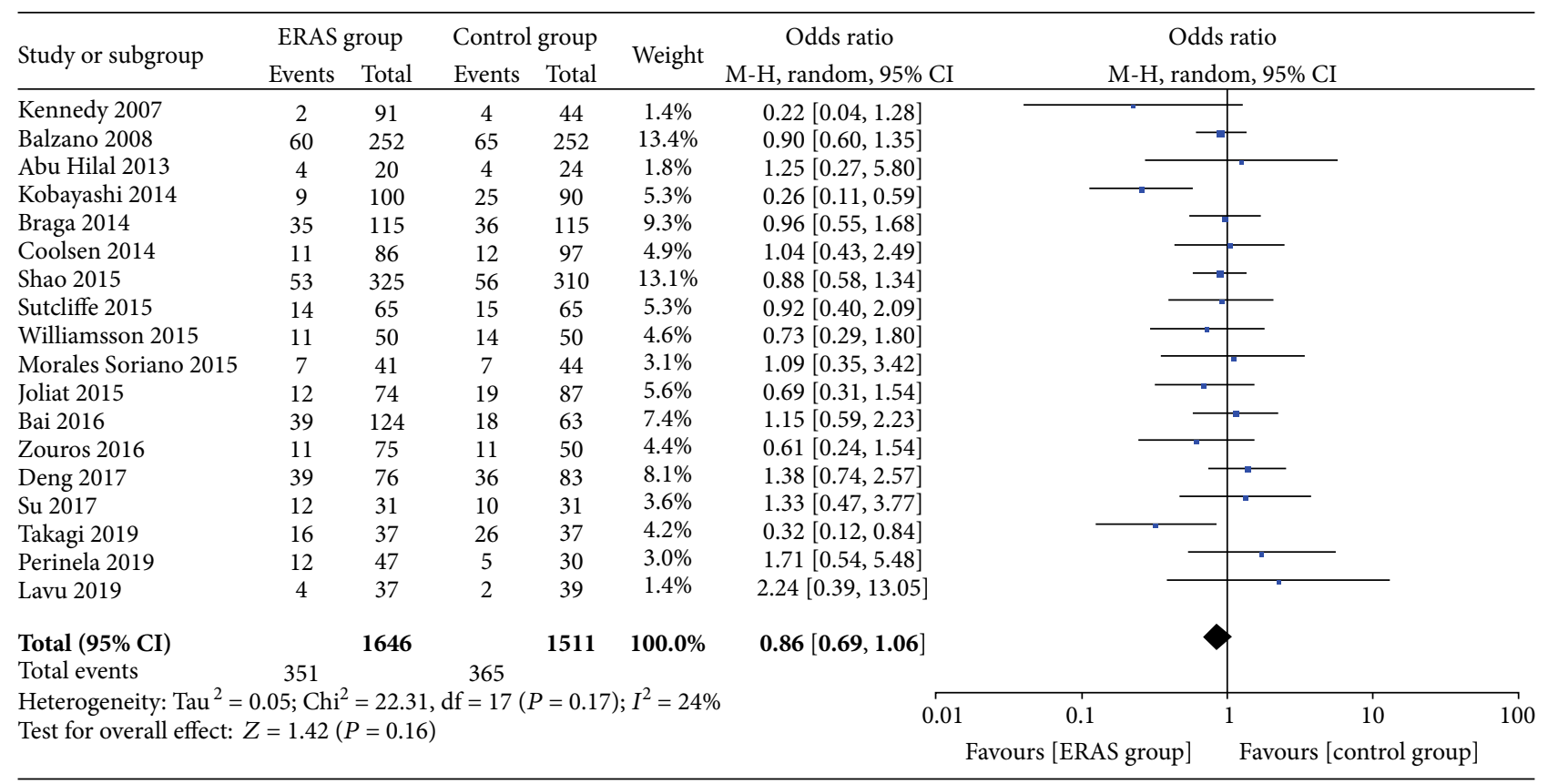

FIGURE 4: Forest plots demonstrating the outcomes of pancreatic fistulas.

assessment form for the included studies is shown in Table S4.

\subsection{Meta-Analysis Outcomes}

\subsubsection{Primary Outcome}

(1) Overall Postoperative Complications. A total of 18 studies [19-32, 34-36, 38] reported the incidence of overall postoperative complications. The meta-analysis results showed that the rate of overall postoperative complications was significantly lower in the ERAS group (OR $=0.62,95 \% \mathrm{CI}$ : 0.53 $0.74, P<0.00001 ;$ Figure 2). In addition, 13 studies [20, $22,24,25,27,28,30-32,34-36,38]$ classified the incidence of overall postoperative complications based on the ClavienDindo severity definitions. The incidence of minor complications (Clavien-Dindo I-II) was lower in the ERAS group than in the control group $(\mathrm{OR}=0.70,95 \% \mathrm{CI}$ : $0.58-0.86$, $P=0.0005)$; however, there were no statistically significant differences in the moderate and severe complications (Clavien-Dindo III-IV; OR $=1.06,95 \% \mathrm{CI}=0.80-1.41, P=$ 0.69 ; Figure 3) between the two groups.

\subsection{Secondary Outcomes}

3.5.1. Pancreatic Fistula. A total of 18 studies [19, 21-37] also reported the incidence of pancreatic fistula. The metaanalysis showed no significant difference between the two groups (OR $=0.86,95 \% \mathrm{CI}$ : 0.69-1.06, $P=0.16$; Figure 4). Furthermore, 13 studies [21, 24, 25, 27-29, 31-37] subdivided the severity of pancreatic fistulas according to the pancreatic fistula grading standard $(\mathrm{A} / \mathrm{B} / \mathrm{C})$ developed by the International Pancreatic Fistula Study Group (ISGPF). The combined analysis also demonstrated that there were no significant differences in Grade A (OR $=0.92$, 95\% CI: 0.68 -
$1.25, P=0.61)$, Grade B (OR $=0.99,95 \% \mathrm{CI}: 0.73-1.33, P=$ 0.94), and Grade C (OR =0.90, 95\% CI: 0.63-1.29, $P=0.57$; Figure 5) pancreatic fistulas between the two groups.

3.5.2. Delayed Gastric Emptying. A total of 18 studies [19, 2137] reported the incidence of delayed gastric emptying, and a total of 3157 patients were involved. Compared to the control group, the ERAS group had a significantly lower incidence of delayed gastric emptying $(\mathrm{OR}=0.51,95 \% \mathrm{CI}: 0.42-0.63$, $P<0.00001$; Figure 6)

3.5.3. Length of Hospital Stay. Eight studies [23, 24, 26, 29, $31-33,35]$ reported the length of hospital stay, and 1685 patients were involved. The current results revealed that the ERAS group had a significantly shorter length of hospital stay than the control group (WMD $=-4.27,95 \%$ CI: $-4.81 \sim-3.73$, $P<0.00001$; Figure 7).

3.5.4. Other Outcomes. The rate of wound infections (OR $=0.82$, 95\% CI: 0.53-1.26, $P=0.36$; Figure 8 ), the rate of abdominal abscesses (OR $=0.91,95 \% \mathrm{CI}$ : 0.64-1.29, $P=$ 0.59; Figure 9), readmission rates $(\mathrm{OR}=1.04,95 \% \mathrm{CI}$ : 0.82 $1.33, P=0.75$; Figure 10$)$, reoperation rates $(\mathrm{OR}=1.04$, 95\% CI: $0.73-1.49, P=0.81$; Figure 11$)$, and morbidity rates (OR $=0.77,95 \%$ CI: 0.55-1.07, $P=0.12$; Figure 12) were not significantly different between the two groups.

3.5.5. Subgroup Analysis and Sensitivity Analysis. Subgroup analysis was performed by separately analyzing only Western countries [19-22, 24, 25, 27-30, 32, 36, 37], Eastern countries $[23,26,31,33-35,38]$, randomized controlled trials $[33,35$, $37,38]$, and case-control studies [19-32, 34, 36]. All of the subgroups produced outcomes consistent with the overall outcomes, except the RCT subgroup. We considered that the main reason contributing to the difference was an 


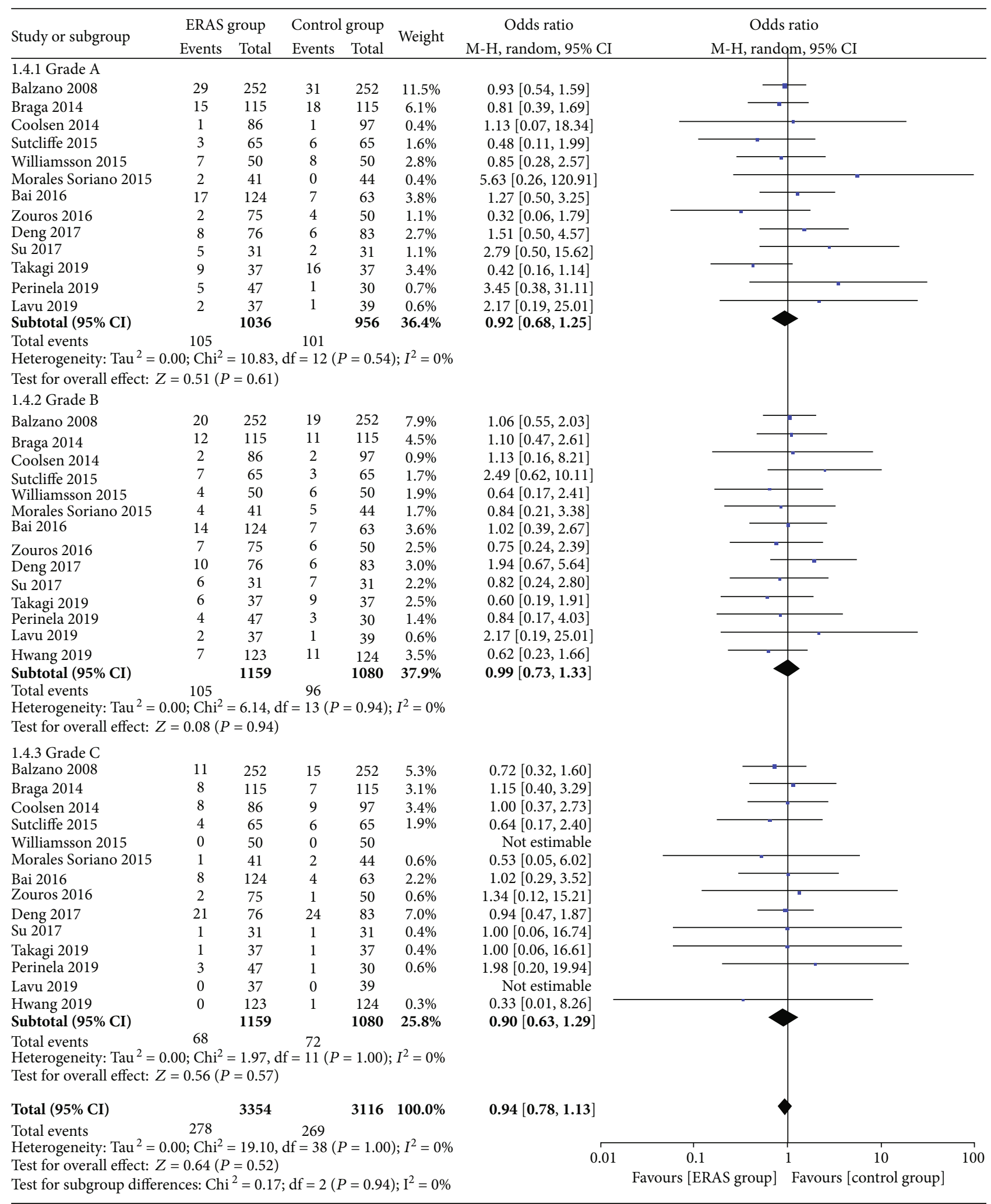

FIgURE 5: Forest plots demonstrating the outcomes of pancreatic fistula according to the definition of International Pancreatic Fistula Study Group.

insufficient number of RCTs. When the analysis focused only on Western countries, the heterogeneity between studies dropped dramatically or even disappeared. However, among Eastern countries, the heterogeneity was obviously increased compared to the overall results. When only the case-control studies were analyzed, both the outcomes and 


\begin{tabular}{|c|c|c|c|c|c|c|c|c|c|}
\hline \multirow{2}{*}{ Study or subgroup } & \multicolumn{2}{|c|}{ ERAS group } & \multicolumn{2}{|c|}{ Control group } & \multirow{2}{*}{ Weight } & \multirow{2}{*}{$\begin{array}{l}\text { Odds ratio } \\
\mathrm{M}-\mathrm{H} \text {, random, } 95 \% \mathrm{CI}\end{array}$} & \multirow{2}{*}{\multicolumn{2}{|c|}{$\begin{array}{c}\text { Odds ratio } \\
\mathrm{M}-\mathrm{H}, \text { random, } 95 \% \mathrm{CI}\end{array}$}} & \\
\hline & Events & Total & Events & Total & & & & & \\
\hline Kennedy 2007 & 7 & 91 & 3 & 44 & $2.2 \%$ & $1.14[0.28,4.63]$ & & & \\
\hline Balzano 2008 & 35 & 252 & 62 & 252 & $20.3 \%$ & $0.49[0.31,0.78]$ & -- & & \\
\hline Abu Hilal 2013 & 1 & 20 & 2 & 24 & $0.7 \%$ & $0.58[0.05,6.90]$ & & & \\
\hline Kobayashi 2014 & 2 & 100 & 9 & 90 & $1.7 \%$ & $0.18[0.04,0.87]$ & & & \\
\hline Braga 2014 & 11 & 115 & 17 & 115 & $6.5 \%$ & $0.61[0.27,1.37]$ & & & \\
\hline Coolsen 2014 & 11 & 86 & 7 & 97 & $4.3 \%$ & $1.89[0.70,5.11]$ & & & \\
\hline Shao 2015 & 29 & 325 & 52 & 310 & $18.1 \%$ & $0.49[0.30,0.79]$ & & & \\
\hline Sutcliffe 2015 & 2 & 65 & 3 & 65 & $1.3 \%$ & $0.66[0.11,4.06]$ & & & \\
\hline Williamsson 2015 & 13 & 50 & 24 & 50 & $6.0 \%$ & $0.38[0.16,0.88]$ & & & \\
\hline Morales Soriano 2015 & 1 & 41 & 3 & 44 & $0.8 \%$ & $0.34[0.03,3.42]$ & & & \\
\hline Joliat 2015 & 20 & 74 & 29 & 87 & $9.2 \%$ & $0.74[0.38,1.46]$ & & - & \\
\hline Bai 2016 & 11 & 124 & 10 & 63 & $5.1 \%$ & $0.52[0.21,1.29]$ & & & \\
\hline Zouros 2016 & 9 & 75 & 15 & 50 & $5.0 \%$ & $0.32[0.13,0.80]$ & & & \\
\hline Deng 2017 & 15 & 76 & 32 & 83 & $8.3 \%$ & $0.39[0.19,0.80]$ & & & \\
\hline Su 2017 & 2 & 31 & 7 & 31 & $1.5 \%$ & $0.24[0.04,1.25]$ & & & \\
\hline Takagi 2019 & 5 & 37 & 7 & 37 & $2.7 \%$ & $0.67[0.19,2.34]$ & & & \\
\hline Perinela 2019 & 7 & 47 & 7 & 30 & $3.1 \%$ & $0.57[0.18,1.85]$ & & & \\
\hline Lavu 2019 & 5 & 37 & 13 & 39 & $3.2 \%$ & $0.31[0.10,0.99]$ & & & \\
\hline Total $(95 \% \mathrm{CI})$ & & 1646 & & 1511 & $100.0 \%$ & $0.51[0.42,0.63]$ & & & \\
\hline Total events & 186 & & 302 & & & & & & \\
\hline \multirow{2}{*}{\multicolumn{6}{|c|}{$\begin{array}{l}\text { Heterogeneity: } \mathrm{Tau}^{2}=0.00 ; \mathrm{Chi}^{2}=14.85, \mathrm{df}=17(P=0.61) ; I^{2}=0 \% \\
\text { Test for overall effect: } Z=6.32(P<0.00001)\end{array}$}} & \multirow[t]{2}{*}{0.01} & 0.1 & 1 & 10 \\
\hline & & & & & & & Favours [ERAS grou & Favours & ntrol g \\
\hline
\end{tabular}

FIGURE 6: Forest plots demonstrating the outcomes of delayed gastric emptying.

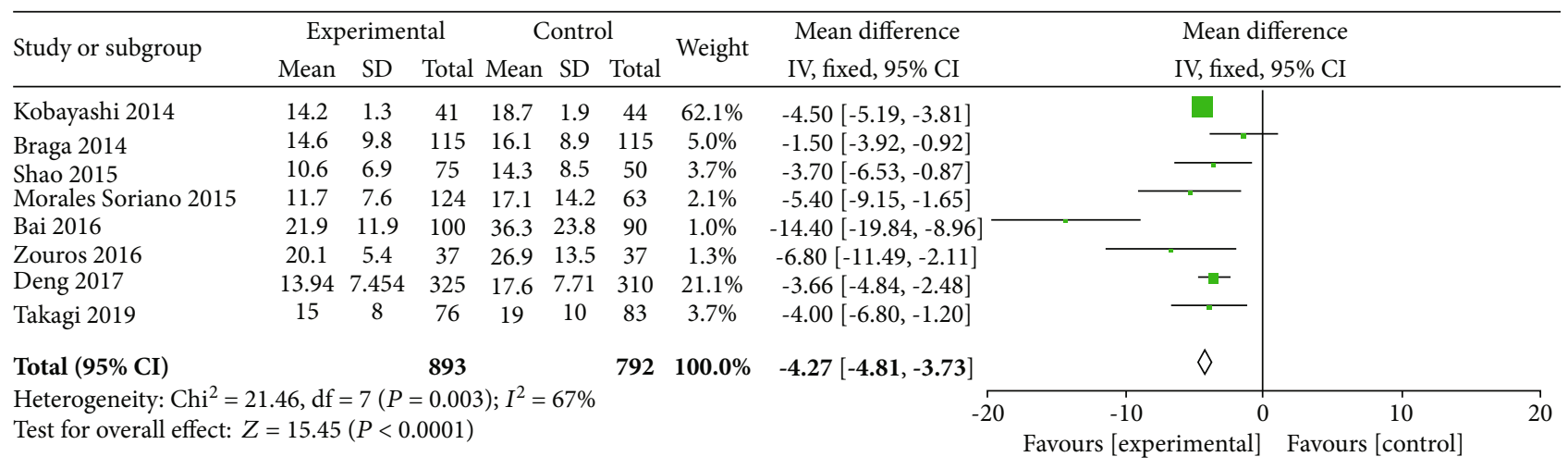

FIgURE 7: Forest plots demonstrating the outcomes of length of hospital stay.

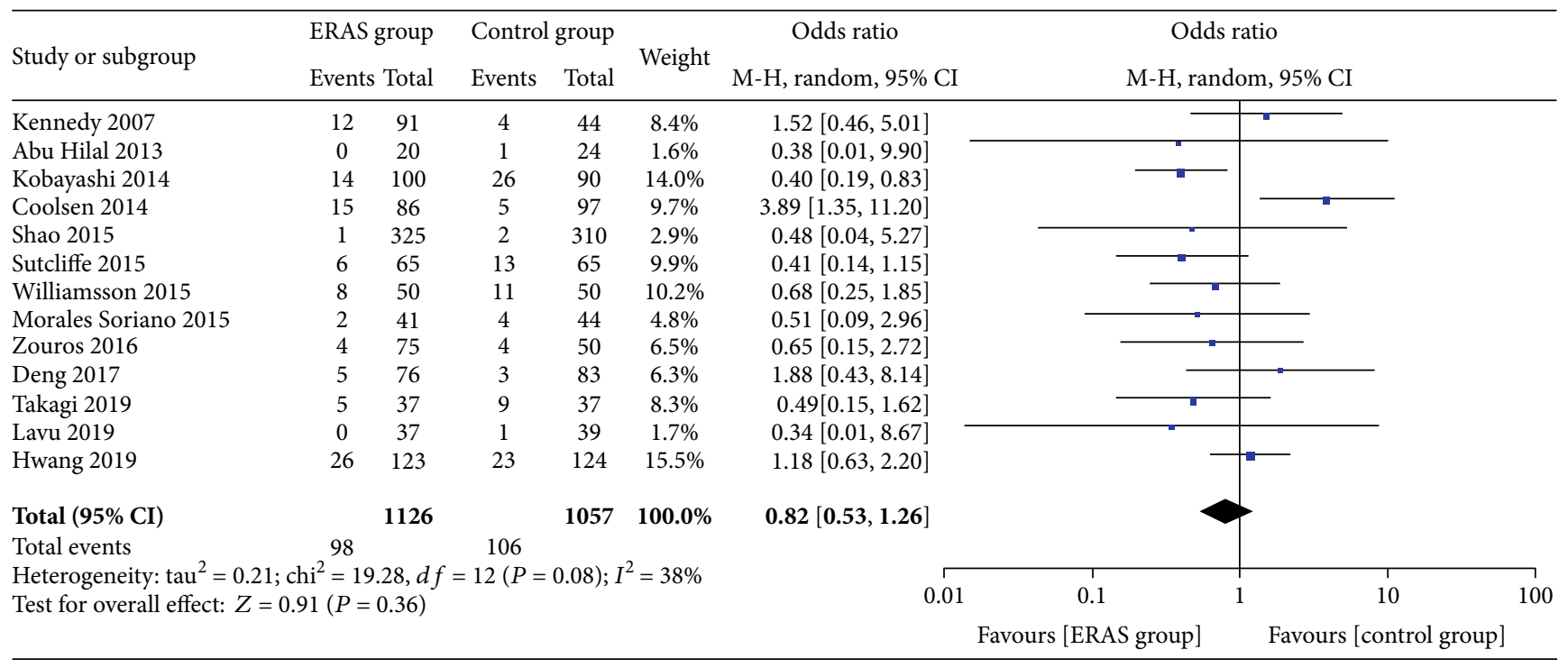

FIGURE 8: Forest plots demonstrating the outcomes of wound infection. 


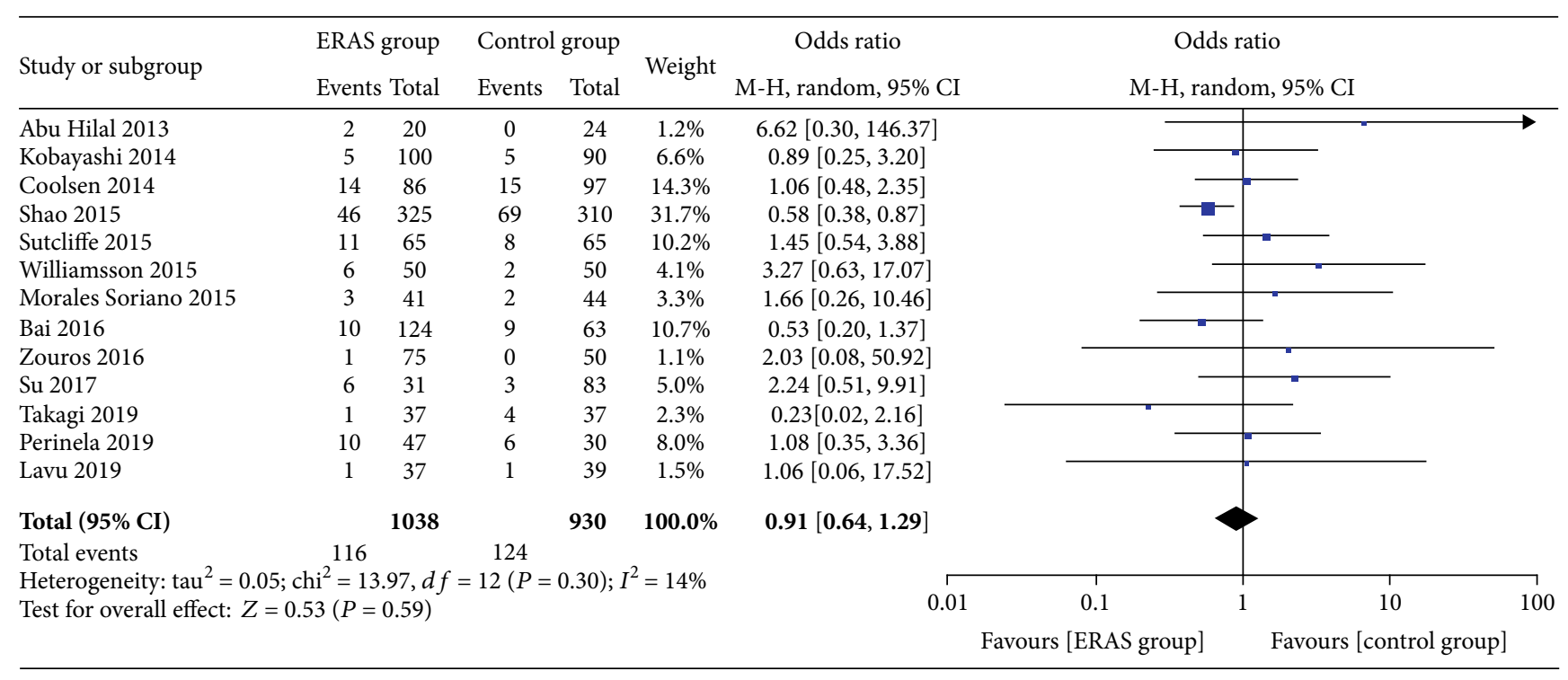

FIGURE 9: Forest plots demonstrating the outcomes of abdominal abscesses.

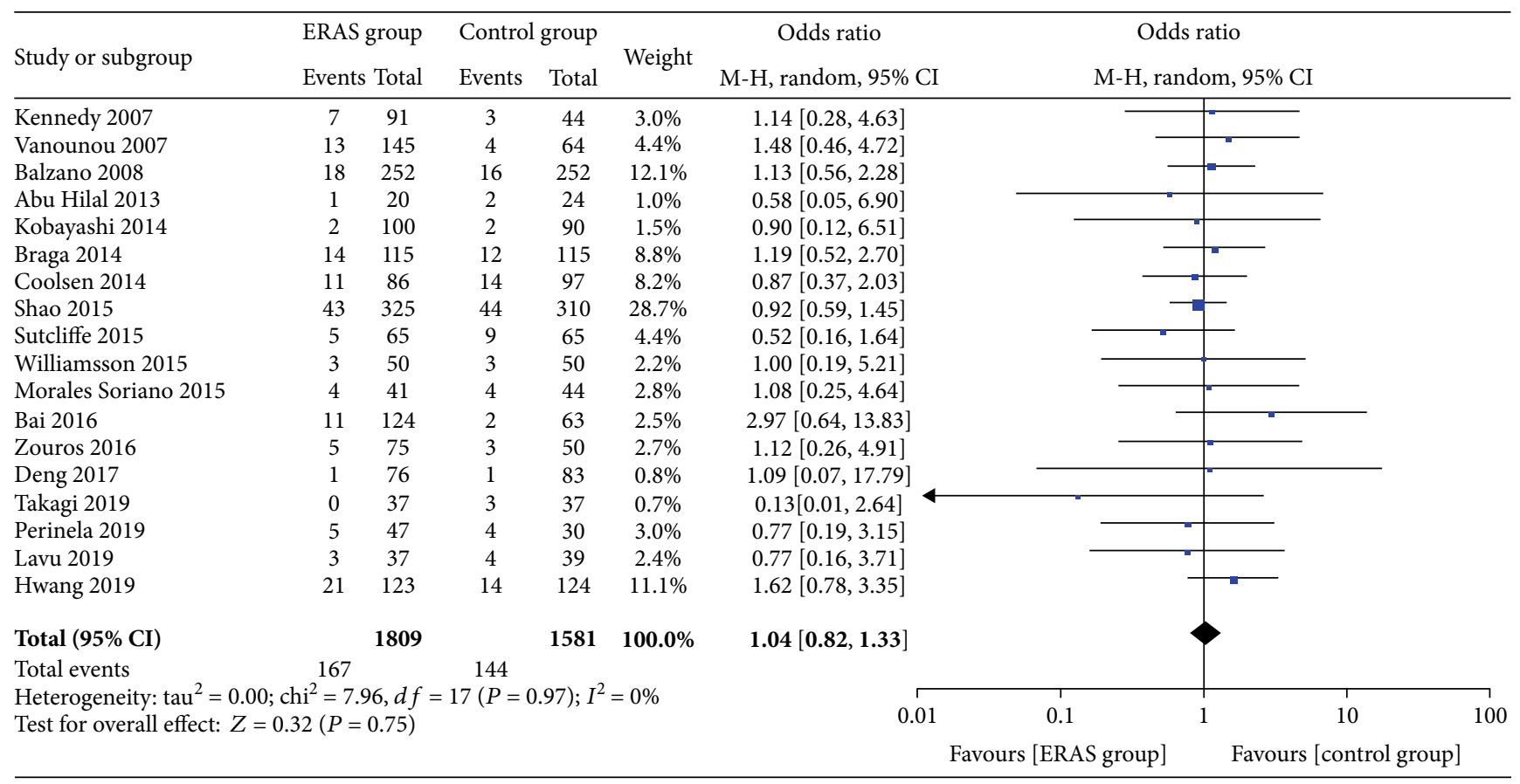

FIGURE 10: Forest plots demonstrating the outcomes of readmission.

the heterogeneity of each subgroup were very close to the overall results. All of the results of the subgroup analyses are displayed in Table 2.

The overall postoperative complications were taken as the outcome, and sensitivity analyses were carried out by excluding one study in each round. Sensitivity analysis showed that no knockout of every study had a particularly large effect on the results. It is worth mentioning that when the study of Coolsen et al. [25] was removed, the heterogeneity was eliminated. All of the results of the sensitivity analyses are presented in Table S5.
3.5.6. Publication Bias. The incidence of overall postoperative complications, pancreatic fistulas, and delayed gastric emptying and mortality rates were drawn as funnel plots to evaluate the potential publication bias. The funnel plots indicated a left-right symmetrical distribution, and publication bias had little impact on the meta-analysis (Figure 13).

\section{Discussion}

Pancreaticoduodenectomy is the main or perhaps the only potentially curative treatment for malignant tumors of the 


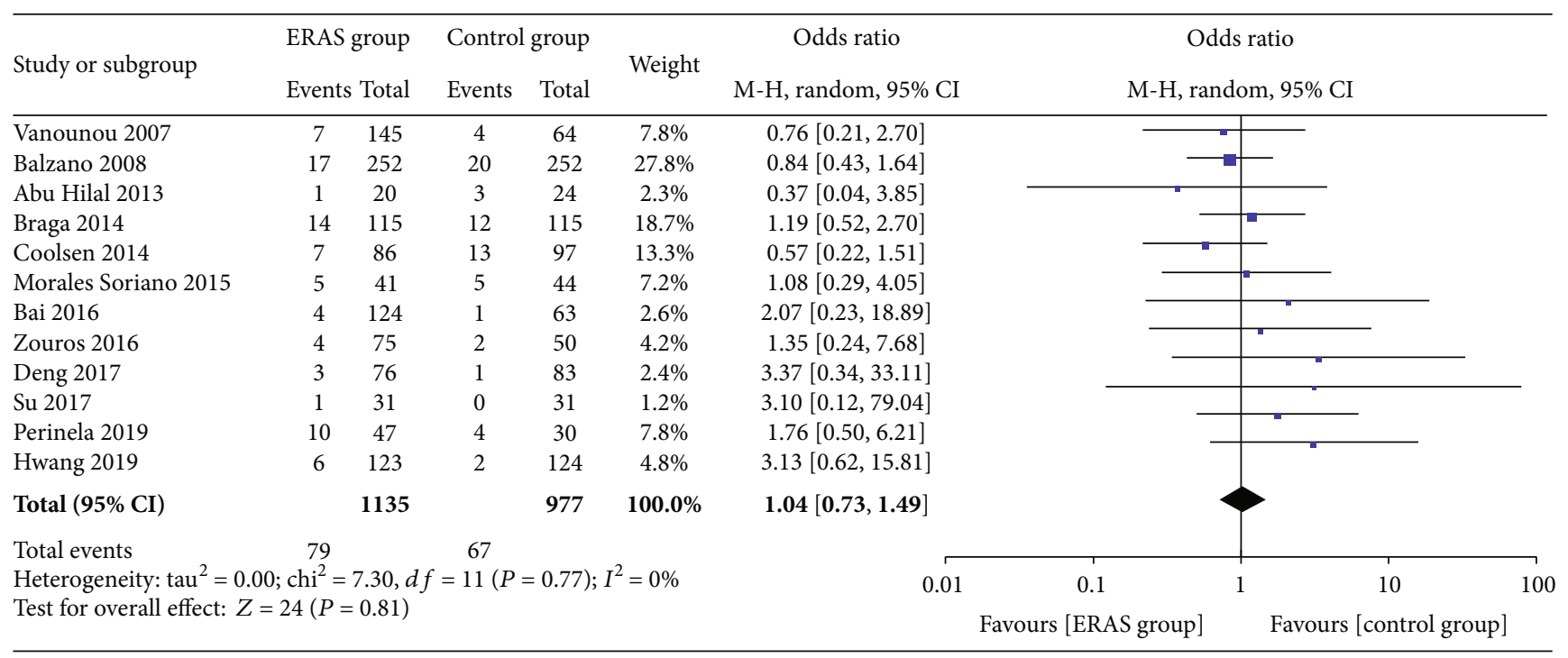

FIGURE 11: Forest plots demonstrating the outcomes of reoperation.

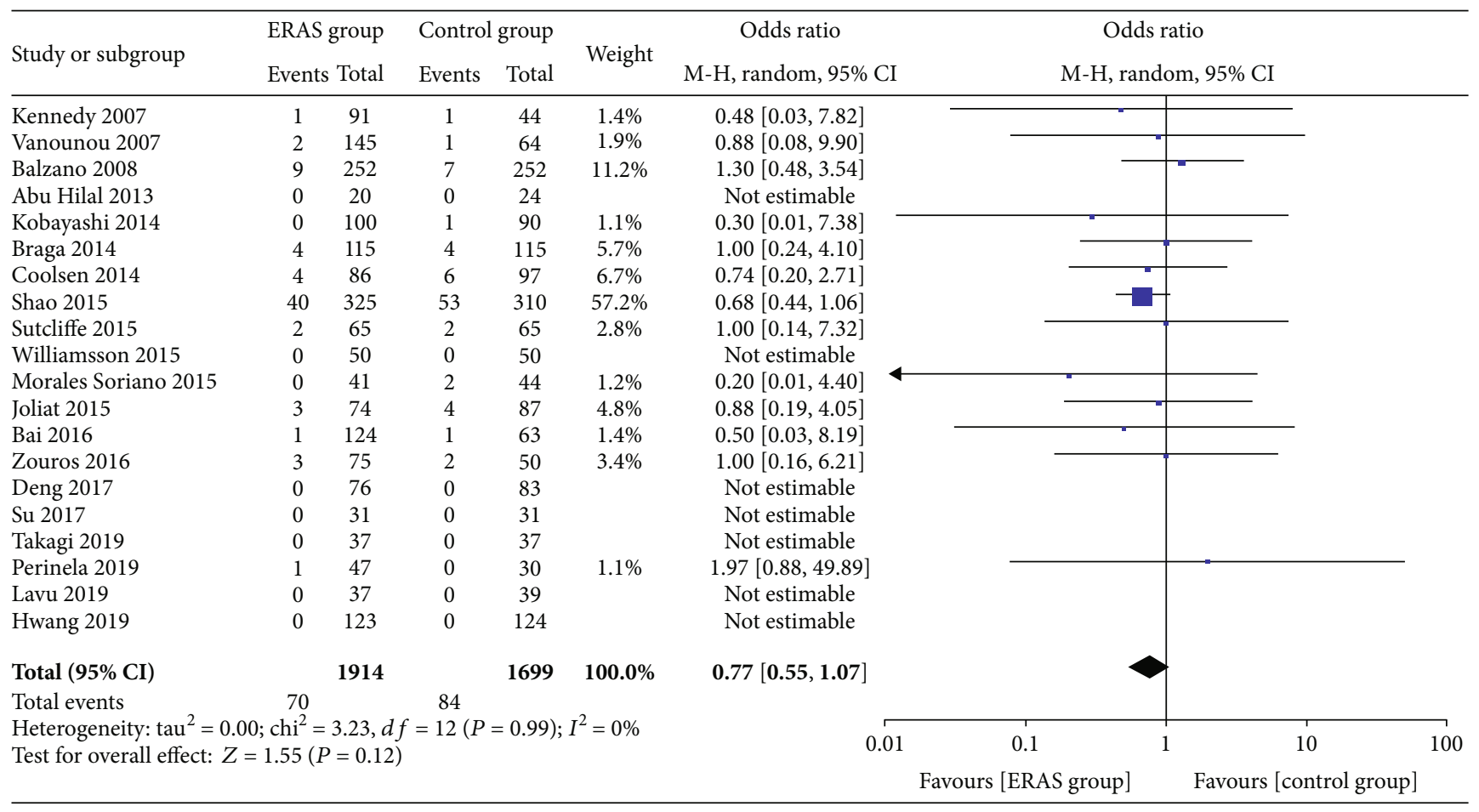

FIGURE 12: Forest plots demonstrating the outcomes of morbidity.

pancreas and duodenal ampulla. However, pancreaticoduodenectomy is complicated and has a high rate of postoperative complications. With the development of medical technology, the introduction of various advanced instruments and equipment, the continuous optimization of perioperative management, and the tendency for centralization in pancreaticoduodenectomy, the mortality rate is approximately 5\% $[39,40]$, and even in some high-volume centers, the mortality rate has even been reduced to $1-2 \%[41,42]$. However, the rate of overall postoperative complications is still as high as 30\%-60\% [43-45]. In particular, complications such as pancreatic fistula, delayed gastric emptying, wound infections, and abdominal abscesses prolong the length of hospital stay and increase the risk for readmission, reoperation, and even death. Therefore, higher requirements need to be put forward for more refined perioperative management in the clinic. The ERAS is safe and has been effectively implemented in colorectal surgery; currently, the approach has been widely accepted and internationally applied to patients undergoing pancreaticoduodenectomy. 
TABLE 2: Results of subgroup analysis.

\begin{tabular}{|c|c|c|c|c|c|c|c|}
\hline Outcome of interest & No. of studies & No. of patients & OR/WMD & $95 \% \mathrm{CI}$ & $P$ value & Heterogeneity $P$ value & $I^{2} \%$ \\
\hline \multicolumn{8}{|c|}{ Studies in Western countries } \\
\hline $\mathrm{PF}$ & 12 & 1850 & 0.89 & $0.71-1.13$ & 0.35 & 0.85 & 0 \\
\hline DGE & 12 & 1850 & 0.56 & $0.43-0.73$ & $<0.0001$ & 0.43 & 1 \\
\hline Overall morbidity & 12 & 1983 & 0.68 & $0.56-0.82$ & $<0.0001$ & 0.46 & 0 \\
\hline LOS & 3 & 491 & -3.30 & $-5.17,-1.44$ & 0.0005 & 0.06 & 63 \\
\hline Readmission & 12 & 1898 & 0.99 & $0.72-1.38$ & 0.97 & 1.00 & 0 \\
\hline Reoperation & 8 & 1457 & 0.92 & $0.63-1.34$ & 0.67 & 0.86 & 0 \\
\hline Mortality & 13 & 2059 & 0.95 & $0.56-1.61$ & 0.84 & 0.99 & 0 \\
\hline \multicolumn{8}{|c|}{ Studies in Eastern countries } \\
\hline $\mathrm{PF}$ & 6 & 1307 & 0.76 & $0.46-1.27$ & 0.30 & 0.008 & 68 \\
\hline DGE & 6 & 1307 & 0.44 & $0.32-0.62$ & $<0.00001$ & 0.77 & 0 \\
\hline Overall morbidity & 6 & 1395 & 0.54 & $0.39-0.74$ & $<0.00001$ & 0.17 & 36 \\
\hline LOS & 5 & 1194 & -4.36 & $-4.93,-3.79$ & $<0.00001$ & 0.005 & 73 \\
\hline Readmission & 6 & 1492 & 1.12 & $0.75-1.66$ & 0.58 & 0.39 & 4 \\
\hline Reoperation & 4 & 655 & 2.88 & $0.99-8.41$ & 0.05 & 0.99 & 0 \\
\hline Mortality & 7 & 1554 & 0.67 & $0.43-1.03$ & 0.07 & 0.86 & 0 \\
\hline \multicolumn{8}{|l|}{ Case-control studies } \\
\hline $\mathrm{PF}$ & 15 & 2848 & 0.85 & $0.70-1.04$ & 0.11 & 0.38 & 7 \\
\hline DGE & 15 & 2848 & 0.53 & $0.43-0.61$ & $<0.0001$ & 0.50 & 0 \\
\hline Overall morbidity & 16 & 3057 & 0.62 & $0.52-0.73$ & $<0.00001$ & 0.31 & 13 \\
\hline LOS & 6 & 891 & -4.46 & $-5.08,-3.83$ & $<0.00001$ & 0.001 & 75 \\
\hline Readmission & 14 & 2834 & 1.01 & $0.77-1.31$ & 0.96 & 0.98 & 0 \\
\hline Reoperation & 10 & 1706 & 0.96 & $0.66-1.38$ & 0.82 & 0.89 & 0 \\
\hline Mortality & 16 & 3057 & 0.77 & $0.55-1.07$ & 0.12 & 0.99 & 0 \\
\hline \multicolumn{8}{|l|}{ RCT } \\
\hline $\mathrm{PF}$ & 3 & 309 & 0.91 & $0.30-2.79$ & 0.87 & 0.03 & 72 \\
\hline DGE & 3 & 309 & 0.41 & $0.24-0.71$ & 0.002 & 0.66 & 0 \\
\hline Overall morbidity & 2 & 321 & 0.52 & $0.17-1.62$ & 0.26 & 0.07 & 71 \\
\hline LOS & 2 & 794 & -3.71 & $-4.80,-2.62$ & $<0.00001$ & 0.83 & 0 \\
\hline Readmission & 4 & 556 & 1.24 & $0.64-2.39$ & 0.52 & 0.38 & 2 \\
\hline Reoperation & 2 & 406 & 3.21 & $0.86-12.03$ & 0.08 & 0.96 & 0 \\
\hline
\end{tabular}

OR: odds ratio; WMD: weighted mean difference; CI: confidence interval; PF: pancreatic fistula; DGE: delayed gastric emptying; LOS: length of hospital stay.

ERAS is a multimodal, multidisciplinary, evidence-based approach to care for surgical patients and aims at optimizing the perioperative management and outcomes. The core of the ERAS concept is to reduce the patient's fasting time, provide preoperative treatment with carbohydrates, provide multimodal analgesia, administer goal-directed fluid therapy, promote early feeding, promote early extubation, and implement early mobilization to alleviate the patient's surgical stress responses, reduce postoperative complications, promote function recovery, shorten the length of hospital stay, and achieve rapid recovery. ERAS requires multidisciplinary cooperation among the departments of surgery, anesthesia, nursing, nutrition, pain, and rehabilitation.

Our meta-analysis included the latest 20 studies [19-38] from 2007 to 2019 to evaluate the safety and efficacy of ERAS in patients undergoing pancreaticoduodenectomy. Compared with the previous meta-analysis [13, 14], our study covered more randomized controlled trials, thus conferring a higher grade of medical evidence to support the outcomes. In our study, the baseline characteristics of the ERAS group and control group were consistent, and strict inclusion and exclusion criteria were followed. All of the studies elaborated on standardized definitions for the outcomes; there was no or little heterogeneity in the observation of most outcomes, and the subgroup analysis and sensitivity analysis also had no effect on the outcomes. Our study shows that ERAS can reduce the overall postoperative complication rates, particularly with respect to the minor complication rate, reduce the incidence of DGE, and shorten the length of hospital stay. The incidence of moderate and serious complications, incidence of pancreatic fistula (regardless of Grade $\mathrm{A} / \mathrm{B} / \mathrm{C}$ ), incidence of incision infections, incidence of abdominal abscesses, readmission rate, reoperation rates, and mortality rates were not significantly different between the two groups $(P>0.05)$, which were confirmatory of previous analyses $[13,14]$. 


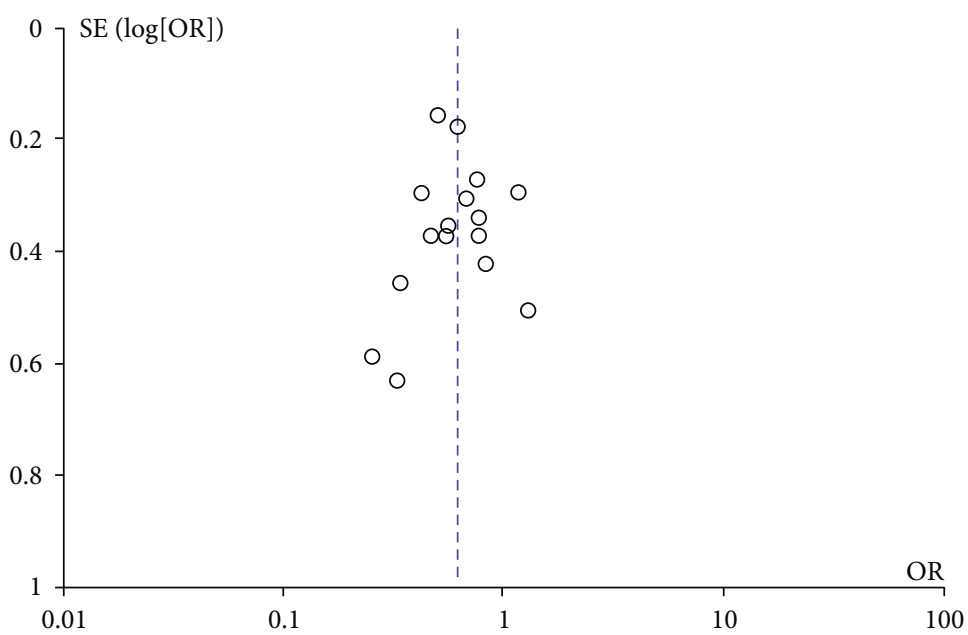

(a)

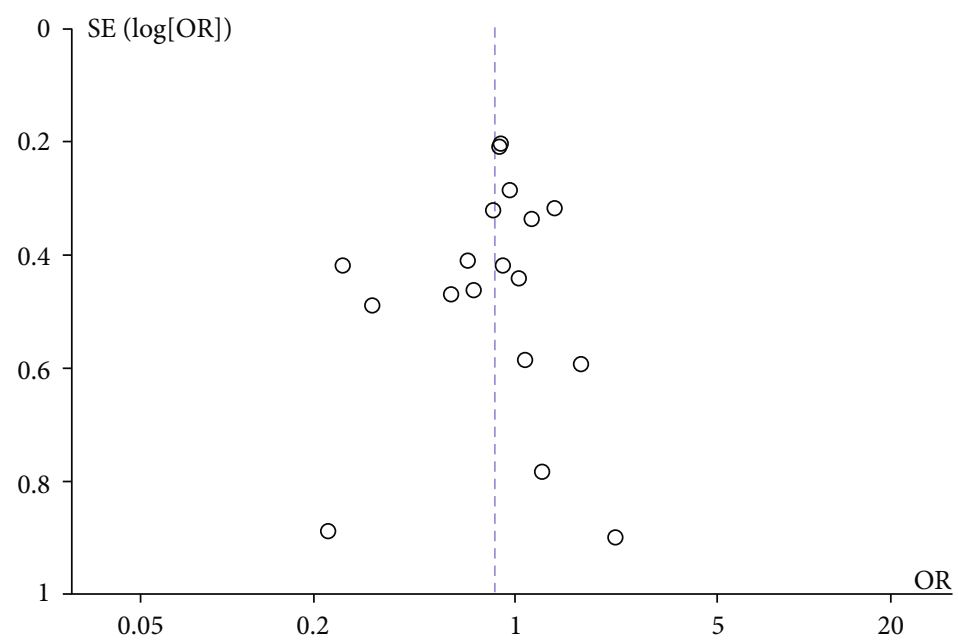

(b)

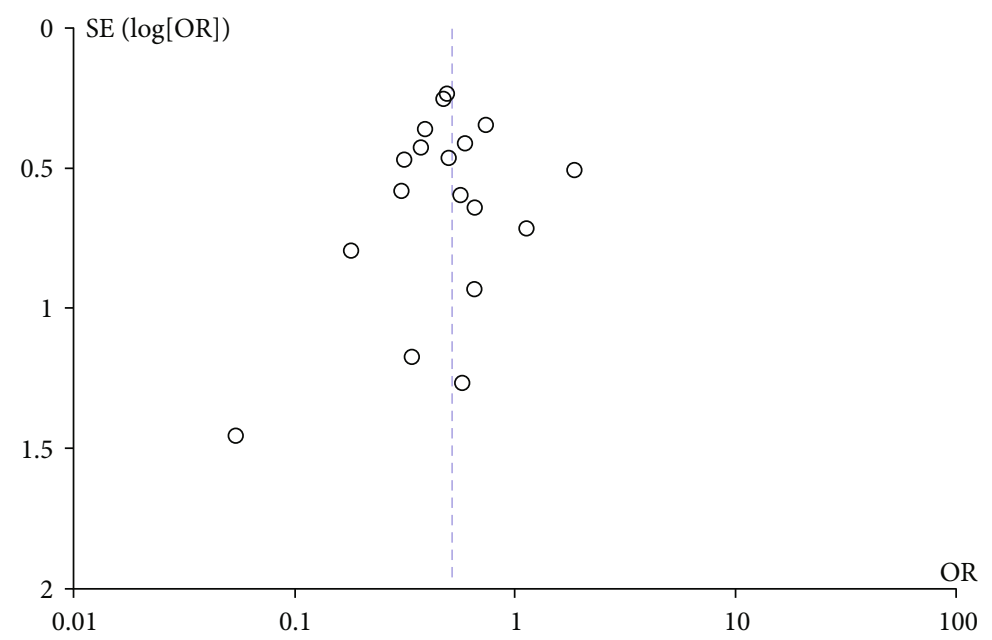

(c)

Figure 13: Continued. 


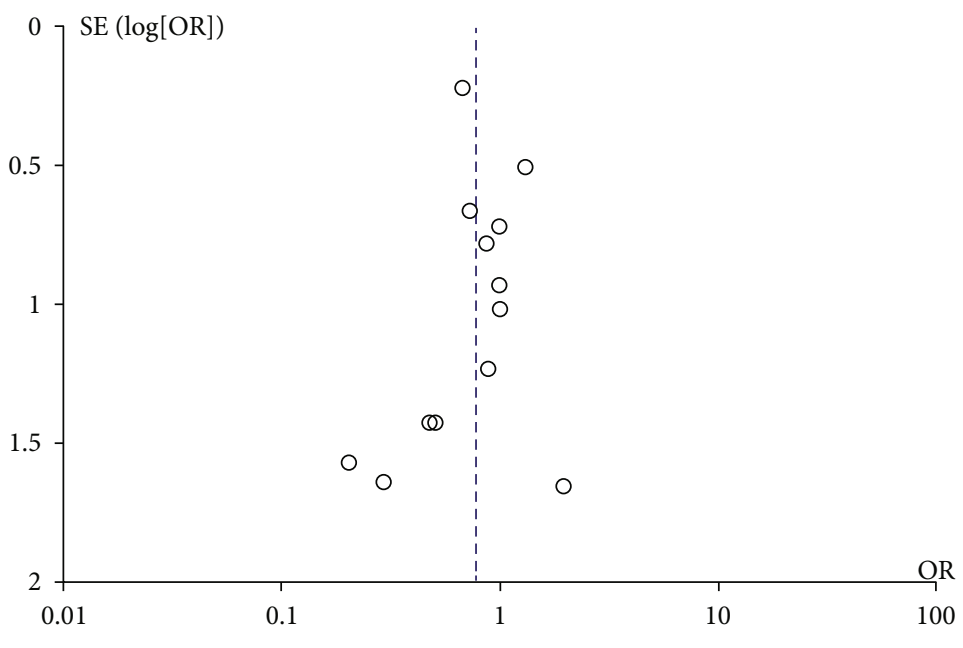

(d)

FIGURE 13: Funnel plot of overall postoperative complications (a), pancreatic fistula (b), delayed gastric emptying (c), and morbidity (d) in all included studies. SE: standard deviation; OR: odds ratio.

Five of the articles $[24,31,32,34,35]$ we included mentioned patient compliance. Braga et al. [24] found that the compliance with preoperative and intraoperative ERAS items was higher (84\%-100\%), while compliance with postoperative ERAS items was relatively low (38\%-66\%). The subgroup analysis showed that better compliance was observed in patients without complications than in those with complications. Furthermore, patient compliance gradually decreased as more severe postoperative complications occurred. Bai et al. [31] demonstrated that the rate of preoperative compliance with ERAS core elements was $74.8 \%-100 \%$; however, the rate of postoperative compliance was $60.4 \%-95.2 \%$. Similarly, Zouros et al. [32] reported that the rate of compliance with various elements ranged from $74.7 \%$ to $100 \%$ and that patients with no complications or minor postoperative complications had a higher adherence rate to ERAS and shorter hospital stay than those with major complications. Su et al. [34] showed that the compliance with preoperative and intraoperative ERAS protocol elements was $71 \%-100 \%$, but the compliance with postoperative was decreased to $58 \%-84 \%$. Takagi et al. [35] found that $84 \%$ of the patients followed the preoperative and intraoperative ERAS protocol, while only $30 \%$ of the patients followed the postoperative ERAS pathway. Wong et al. [46] investigated the implementation of protocols based on the ERAS concept in the perioperative period of liver surgery in 11 HPB centers in Europe, and the results showed that the compliance rate for the postoperative ERAS protocol was not promising. A survey [47] of 2352 colorectal surgery patients who were treated with the ERAS protocol in 13 centers from 6 countries showed that a higher compliance rate was associated with a lower postoperative complication rate and shorter length of hospital stay. Therefore, improvements in patient compliance are essential to guaranteeing that ERAS to be implemented in practice.

Several potential limitations of the present analysis should be acknowledged. First, the majority of the studies included were retrospective case-control studies, which may lead to selection bias and recall bias. None of the randomized controlled trials featured blinding for the subjects, intervention operators, or outcome measurers, which may lead to implementation bias and measurement bias. Second, the specific ERAS protocols vary among different studies, and they included a minimum of 9 items and a maximum of 25 items; additionally, patient compliance was hard to control, which may lead to clinical heterogeneity. It is worth mentioning that the implementation of a blinding method for the ERAS protocol itself is not feasible; this is also the main reason why the previous meta-analysis only included case-control studies and the main factor that led to the moderate quality of RCTs in our study.

In conclusion, the ERAS approach is safe and effective in the perioperative management of pancreatoduodenectomy patients, and it can accelerate the postoperative recovery, promote better recovery with respect to gastrointestinal function, and shorten the length of hospital stay. Large-sample, multicenter, prospective research is needed to provide more solid evidence. Currently, the ERAS guidelines in many fields advocate for the selection of minimally invasive surgery, and since laparoscopic pancreaticoduodenectomy is maturing in hepatobiliary surgery, we look forward to more literature that reports the application of ERAS for LPD patients. In clinical practice, an ERAS team is required to ensure that each item is implemented, provide precise and individualized patient management, improve patient compliance, and promote patient recovery.

\section{Data Availability}

The datasets used and/or analyzed during the current study are available from the corresponding author on reasonable request. 


\section{Additional Points}

Core Tip. This is a meta-analysis with the latest 20 studies up to July 2019 that evaluates the safety and efficacy of ERAS in patients undergoing pancreaticoduodenectomy. Compared to the previous meta-analysis that only included casecontrol studies, our study first covered randomized controlled trials and confers a higher grade of medical evidence to support the outcomes. We applied strict inclusion and exclusion criteria and demanded higher standards on the quality of the included studies. In addition, our study elaborated on the standardized definitions of outcomes and comprehensively summarized the perioperative interventions in the ERAS group to ensure that the conclusion is highly reliable.

PRISMA 2009 Checklist Statement. The authors have read the PRISMA 2009 Checklist, and the manuscript was prepared and revised according to the PRISMA 2009 Checklist.

\section{Conflicts of Interest}

There are no conflicts of interest associated with any of authors of this manuscript.

\section{Supplementary Materials}

Table S1: PubMed Search Strategy. Table S2: demographics of the included studies. Table S3: elements of enhanced recovery after surgery. Table S4: bias risk assessment form of the included studies. Table S5: results of sensitivity analysis. (Supplementary Materials)

\section{References}

[1] H. Kehlet, "Multimodal approach to control postoperative pathophysiology and rehabilitation," British Journal of Anaesthesia, vol. 78, no. 5, pp. 606-617, 1997.

[2] H. Kehlet and D. W. Wilmore, "Evidence-based surgical care and the evolution of fast-track surgery," Annals of Surgery, vol. 248, no. 2, pp. 189-198, 2008.

[3] K. C. Fearon, O. Ljungqvist, M. von Meyenfeldt et al., "Enhanced recovery after surgery: a consensus review of clinical care for patients undergoing colonic resection," Clinical Nutrition, vol. 24, no. 3, pp. 466-477, 2005.

[4] J. Nygren, J. Thacker, F. Carli et al., "Guidelines for perioperative care in elective rectal/pelvic surgery: Enhanced Recovery After Surgery (ERAS(R)) Society recommendations," Clinical Nutrition, vol. 31, no. 6, pp. 801-816, 2012.

[5] K. Lassen, M. M. Coolsen, K. Slim et al., "Guidelines for perioperative care for pancreaticoduodenectomy: Enhanced Recovery After Surgery (ERAS(R)) Society recommendations," Clinical Nutrition, vol. 31, no. 6, pp. 817-830, 2012.

[6] Y. Cerantola, M. Valerio, B. Persson et al., "Guidelines for perioperative care after radical cystectomy for bladder cancer: Enhanced Recovery After Surgery (ERAS ${ }^{\circledR}$ ) society recommendations," Clinical Nutrition, vol. 32, no. 6, pp. 879-887, 2013.

[7] K. Mortensen, M. Nilsson, K. Slim et al., "Consensus guidelines for enhanced recovery after gastrectomy: Enhanced Recovery After Surgery (ERAS(R)) Society recommenda- tions," The British Journal of Surgery, vol. 101, no. 10, pp. 1209-1229, 2014.

[8] T. J. P. Batchelor, N. J. Rasburn, E. Abdelnour-Berchtold et al., "Guidelines for enhanced recovery after lung surgery: recommendations of the Enhanced Recovery After Surgery (ERAS(R)) Society and the European Society of Thoracic Surgeons (ESTS)," European Journal of Cardio-Thoracic Surgery, vol. 55, no. 1, pp. 91-115, 2019.

[9] U. O. Gustafsson, M. J. Scott, M. Hubner et al., "Guidelines for perioperative care in elective colorectal surgery: Enhanced Recovery After Surgery (ERAS((R))) Society recommendations: 2018," World Journal of Surgery, vol. 43, no. 3, pp. 659-695, 2019.

[10] G. Nelson, J. Bakkum-Gamez, E. Kalogera et al., “Guidelines for perioperative care in gynecologic/oncology: Enhanced Recovery After Surgery (ERAS) Society recommendations2019 update," International Journal of Gynecological Cancer, vol. 29, no. 4, pp. 651-668, 2019.

[11] G. A. Macones, A. B. Caughey, S. L. Wood et al., "Guidelines for postoperative care in cesarean delivery: Enhanced Recovery After Surgery (ERAS) Society recommendations (part 3)," American Journal of Obstetrics and Gynecology, vol. 221, no. 3, pp. 247.e1-247.e9, 2019.

[12] D. T. Engelman, W. Ben Ali, J. B. Williams et al., "Guidelines for perioperative care in cardiac Surgery," JAMA Surgery, vol. 154, no. 8, p. 755, 2019.

[13] J. Xiong, P. Szatmary, W. Huang et al., "Enhanced Recovery After Surgery program in patients undergoing pancreaticoduodenectomy: a PRISMA-compliant systematic review and meta-analysis," Medicine (Baltimore), vol. 95, no. 18, p. e3497, 2016.

[14] H. B. Ji, W. T. Zhu, Q. Wei, X. X. Wang, H. B. Wang, and Q. P. Chen, "Impact of enhanced recovery after surgery programs on pancreatic surgery: a meta-analysis," World Journal of Gastroenterology, vol. 24, no. 15, pp. 1666-1678, 2018.

[15] K. Slim, E. Nini, D. Forestier, F. Kwiatkowski, Y. Panis, and J. Chipponi, "Methodological index for non-randomized studies (minors): development and validation of a new instrument," ANZ Journal of Surgery, vol. 73, no. 9, pp. 712-716, 2003.

[16] C. Bassi, C. Dervenis, G. Butturini et al., "Postoperative pancreatic fistula: an international study group (ISGPF) definition," Surgery, vol. 138, no. 1, pp. 8-13, 2005.

[17] M. N. Wente, C. Bassi, C. Dervenis et al., "Delayed gastric emptying (DGE) after pancreatic surgery: a suggested definition by the International Study Group of Pancreatic Surgery (ISGPS)," Surgery, vol. 142, no. 5, pp. 761-768, 2007.

[18] D. Dindo, N. Demartines, and P. A. Clavien, "Classification of surgical complications: a new proposal with evaluation in a cohort of 6336 patients and results of a survey," Annals of Surgery, vol. 240, no. 2, pp. 205-213, 2004.

[19] E. P. Kennedy, E. L. Rosato, P. K. Sauter et al., "Initiation of a Critical Pathway for Pancreaticoduodenectomy at an Academic Institution-the First Step in Multidisciplinary Team Building," Journal of the American College of Surgeons, vol. 204, no. 5, pp. 917-923, 2007, discussion 923-4.

[20] T. Vanounou, W. Pratt, J. E. Fischer, Vollmer CM Jr, and M. P. Callery, "Deviation-based cost modeling: a novel model to evaluate the clinical and economic impact of clinical pathways," Journal of the American College of Surgeons, vol. 204, no. 4, pp. 570-579, 2007. 
[21] G. Balzano, A. Zerbi, M. Braga, S. Rocchetti, A. A. Beneduce, and V. di Carlo, "Fast-track recovery programme after pancreatico- duodenectomy reduces delayed gastric emptying," The British Journal of Surgery, vol. 95, no. 11, pp. 1387-1393, 2008.

[22] M. Abu Hilal, F. di Fabio, A. A. Badran et al., "Implementation of enhanced recovery programme after pancreatoduodenectomy: a single-centre UK pilot study," Pancreatology, vol. 13, no. 1, pp. 58-62, 2013.

[23] S. Kobayashi, R. Ooshima, S. Koizumi et al., "Perioperative care with fast-track management in patients undergoing pancreaticoduodenectomy," World Journal of Surgery, vol. 38, no. 9, pp. 2430-2437, 2014.

[24] M. Braga, N. Pecorelli, R. Ariotti et al., "Enhanced recovery after surgery pathway in patients undergoing pancreaticoduodenectomy," World Journal of Surgery, vol. 38, no. 11, pp. 2960-2966, 2014.

[25] M. M. E. Coolsen, R. M. van Dam, A. Chigharoe, S. W. M. Olde Damink, and C. H. C. Dejong, "Improving outcome after pancreaticoduodenectomy: experiences with implementing an enhanced recovery after surgery (ERAS) program," Digestive Surgery, vol. 31, no. 3, pp. 177-184, 2014.

[26] Z. Shao, G. Jin, W. Ji, L. Shen, and X. Hu, "The role of fasttrack surgery in pancreaticoduodenectomy: a retrospective cohort study of 635 consecutive resections," International Journal of Surgery, vol. 15, pp. 129-133, 2015.

[27] R. P. Sutcliffe, M. Hamoui, J. Isaac et al., "Implementation of an enhanced recovery pathway after pancreaticoduodenectomy in patients with low drain fluid amylase," World Journal of Surgery, vol. 39, no. 8, pp. 2023-2030, 2015.

[28] C. Williamsson, N. Karlsson, C. Sturesson, G. Lindell, R. Andersson, and B. Tingstedt, "Impact of a fast-track surgery programme for pancreaticoduodenectomy," The British Journal of Surgery, vol. 102, no. 9, pp. 1133-1141, 2015.

[29] R. Morales Soriano, N. Esteve Pérez, S. Tejada Gavela et al., "Outcomes of an enhanced recovery after surgery programme for pancreaticoduodenectomy," Cirugía Española, vol. 93, no. 8, pp. 509-515, 2015.

[30] G. R. Joliat, I. Labgaa, D. Petermann et al., "Cost-benefit analysis of an enhanced recovery protocol for pancreaticoduodenectomy," The British Journal of Surgery, vol. 102, no. 13, pp. 1676-1683, 2015.

[31] X. Bai, X. Zhang, F. Lu et al., "The implementation of an enhanced recovery after surgery (ERAS) program following pancreatic surgery in an academic medical center of China," Pancreatology, vol. 16, no. 4, pp. 665-670, 2016.

[32] E. Zouros, T. Liakakos, A. Machairas, P. Patapis, C. Agalianos, and C. Dervenis, "Improvement of gastric emptying by enhanced recovery after pancreaticoduodenectomy," Hepatobiliary \& Pancreatic Diseases International, vol. 15, no. 2, pp. 198-208, 2016.

[33] X. Deng, X. Cheng, Z. Huo et al., "Modified protocol for enhanced recovery after surgery is beneficial for Chinese cancer patients undergoing pancreaticoduodenectomy," Oncotarget, vol. 8, no. 29, pp. 47841-47848, 2017.

[34] W. Su, F. Lu, X. Zhang et al., “A hospital-to-home evaluation of an enhanced recovery protocol for elective pancreaticoduodenectomy in China: a cohort study," Medicine (Baltimore), vol. 96, no. 41, p. e8206, 2017.

[35] K. Takagi, R. Yoshida, T. Yagi et al., "Effect of an enhanced recovery after surgery protocol in patients undergoing pan- creaticoduodenectomy: a randomized controlled trial," Clinical Nutrition, vol. 38, no. 1, pp. 174-181, 2019.

[36] J. Perinel, A. Duclos, C. Payet, Y. Bouffard, J. C. Lifante, and M. Adham, "Impact of enhanced recovery program after surgery in patients undergoing pancreatectomy on postoperative outcomes: a controlled before and after study," Digestive Surgery, vol. 37, no. 1, pp. 47-55, 2020.

[37] H. Lavu, N. McCall, J. M. Winter et al., "Enhancing patient outcomes while containing costs after complex abdominal operation: a randomized controlled trial of the Whipple accelerated recovery pathway," Journal of the American College of Surgeons, vol. 228, no. 4, pp. 415-424, 2019.

[38] D. W. Hwang, H. J. Kim, J. H. Lee et al., "Effect of Enhanced Recovery After Surgery program on pancreaticoduodenectomy: a randomized controlled trial," Journal of HepatoBiliary-Pancreatic Sciences, vol. 26, 2019.

[39] G. A. Gooiker, V. E. P. P. Lemmens, M. G. Besselink et al., "Impact of centralization of pancreatic cancer surgery on resection rates and survival," The British Journal of Surgery, vol. 101, no. 8, pp. 1000-1005, 2014.

[40] M. J. Passeri, E. H. Baker, I. A. Siddiqui et al., "Total compared with partial pancreatectomy for pancreatic adenocarcinoma: assessment of resection margin, readmission rate, and survival from the U.S. National Cancer Database," Current Oncology, vol. 26, no. 3, pp. e346-e356, 2019.

[41] F. Miura, M. Yamamoto, M. Gotoh et al., "Validation of the board certification system for expert surgeons (hepato-biliary-pancreatic field) using the data of the National Clinical Database of Japan: part 2 - pancreatoduodenectomy," Journal of Hepato-Biliary-Pancreatic Sciences, vol. 23, no. 6, pp. 353363, 2016.

[42] R. Yoshioka, H. Yasunaga, K. Hasegawa et al., "Impact of hospital volume on hospital mortality, length of stay and total costs after pancreaticoduodenectomy," The British Journal of Surgery, vol. 101, no. 5, pp. 523-529, 2014.

[43] J. Herrera, C. Zazpe, P. Sánchez, A. Tarifa, I. Eguaras, and J. M. Lera, "Feasibility, morbidity and mortality in two hundred consecutive cases of pancreaticogastrostomy after pancreaticoduodenectomy," Cirugía Española, vol. 97, no. 9, pp. 501-509, 2019.

[44] N. A. van der Gaag, K. Harmsen, W. J. Eshuis, O. R. C. Busch, T. M. van Gulik, and D. J. Gouma, "Pancreatoduodenectomy associated complications influence cancer recurrence and time interval to death," European Journal of Surgical Oncology, vol. 40, no. 5, pp. 551-558, 2014.

[45] S. Aoki, H. Miyata, H. Konno et al., "Risk factors of serious postoperative complications after pancreaticoduodenectomy and risk calculators for predicting postoperative complications: a nationwide study of 17,564 patients in Japan," Journal of Hepato-Biliary-Pancreatic Sciences, vol. 24, no. 5, pp. 243251, 2017.

[46] E. M. Wong-Lun-Hing, R. M. van Dam, L. A. Heijnen et al., "Is current perioperative practice in hepatic surgery based on enhanced recovery after surgery (ERAS) principles?," World Journal of Surgery, vol. 38, no. 5, pp. 1127-1140, 2014.

[47] A. Currie, J. Burch, and J. T. Jenkins, "The impact of enhanced recovery protocol compliance on elective colorectal cancer resection: results from an international registry," Annals of Surgery, 2015. 